\title{
Magnetoelectric effects studied by resonant x-ray diffraction in ferrimagnetic $\mathrm{GaFeO}_{3}$
}

\author{
U. Staub, ${ }^{1}$ Y. Bodenthin, ${ }^{1}$ C. Piamonteze, ${ }^{1}$ S. P. Collins, ${ }^{2}$ S. Koohpayeh, ${ }^{3}$ D. Fort, ${ }^{3}$ and S. W. Lovesey ${ }^{2,4}$ \\ ${ }^{1}$ Swiss Light Source, Paul Scherrer Institut, CH 5232 Villigen PSI, Switzerland \\ ${ }^{2}$ Diamond Light Source Ltd., Oxfordshire OX11 ODE, United Kingdom \\ ${ }^{3}$ Department of Metallurgy and Materials, University of Birmingham, Birmingham B15 2TT, United Kingdom \\ ${ }^{4}$ ISIS Facility, Harwell Science and Innovation Campus, Oxfordshire OX11 0QX, United Kingdom
}

(Received 4 March 2010; published 9 September 2010)

\begin{abstract}
Resonant X-ray Bragg diffraction experiments on space group forbidden reflections of type (0k0) with $k$ odd taken in the vicinity of the $\mathrm{Fe} K$ edge are used to study magnetoelectric effects in $\mathrm{GaFeO}_{3}$. The main intensity of the various spectral features is caused by the E1-E1 transition and can be ascribed to the observation of an electric quadrupole moment, giving different intensities within a Friedel pair. Collecting the magnetic field difference intensity by having the magnetization parallel or antiparallel to the $c$ axis in the ferrimagnetic phase is discussed in the framework of magnetoelectric multipole moments, which are both parity and time odd. The azimuthal angle dependence and the sign change in the magnetic signal when going from $(0 k 0)$ to $(0 \bar{k} 0)$ reflections can be understood by the interference of the electric quadrupole with that of magnetoelectric quadrupole and octupole moments.
\end{abstract}

DOI: 10.1103/PhysRevB.82.104411

PACS number(s): 75.85.+t, 75.25.-j, 75.50.Gg

\section{INTRODUCTION}

Since the discovery of multiferroic materials with electric polarization caused by the electronic degree of freedom, ${ }^{1}$ the magnetoelectric effect has attracted significant interest. In many of these materials, there is a strong relation between the occurrence of ferroelectricity and magnetic phase transitions. Moreover, it has been shown that magnetic fields strongly influence the ferroelectric polarization ${ }^{1,2}$ and that the magnetization chirality ${ }^{3}$ and magnetic structure ${ }^{4}$ can be influenced by the application of electric fields. Despite the fact that these experiments directly probe the magnetoelectric coupling, an atomic understanding of the magnetoelectric effect is still missing in these materials.

A multiferroic order parameter based on the coupling of magnetic moments and electric polarization could be produced by considering the toroidal moment (also called anapole moment), which in its simplest form is $\boldsymbol{\Omega}_{i} \propto \mathbf{S}_{i} \times \mathbf{R}_{i}$ with $\mathbf{S}_{i}$ the spin and $\mathbf{R}_{i}$ the electric dipole moment at the same site. Toroidal domains, which could be represented by a ferro-type order of toroidal moments, can be best observed with optical methods, e.g., second-harmonic generation as nicely demonstrated in $\mathrm{LiCoPO}_{4}{ }^{5}$ The observation of toroidal moments on the atomic level on a given site $i$ is much more challenging. Dichroic x-ray techniques (absorption and scattering) can in principle be used to detect atomiclike magnetoelectric multipole moments, for which the toroidal moment represent the dipole (rank 1). Several studies of that type have already been interpreted with use of magnetoelectric multipole moments such as the magnetoelectric monopole, dipole, and quadrupole. ${ }^{6-10}$ Though there is a direct relation between these multipole moments and the magnetoelectric effect, the disentanglement and determination of these atomiclike multipole moments in multiferroics remains a challenge.

An ideal candidate for a fundamental study of these strange magnetoelectric multipoles is $\mathrm{GaFeO}_{3}$, a magnetoelectric material already studied more than 30 years ago. ${ }^{11-13}$
The material is piezoelectric and crystallizes in the polar space group $P C 2{ }_{1} n$ with lattice constants of approximately, $a=8.75, b=9.40$, and $c=5.08 \AA .{ }^{14} \mathrm{The}^{\mathrm{F} e}{ }^{3+}$ ions in $\mathrm{GaFeO}_{3}$ are (with its half-filled-shell electronic configuration $3 d^{5}$ ) expected to be isotropic in its electron-density distribution with zero orbital magnetic moment. The material is ferrimagnetic with magnetization direction along the crystal $c$ axis below $T_{\mathrm{C}} \approx 210 \mathrm{~K}$ and the transition temperature depends on the $\mathrm{Fe}, \mathrm{Ga}$ intersite mixing. ${ }^{15} \mathrm{X}$-ray magnetic circular dichroism experiments showed that the orbital magnetic moment is not really zero, indicative for a large hybridization of $\mathrm{Fe} 3 d$ states with oxygen $2 p$ orbitals. ${ }^{16}$ Optical and x-ray absorption experiments have been performed on $\mathrm{GaFeO}_{3},{ }^{17,18}$ and were interpreted by theoretical calculations based on atomic magnetoelectric multipole moments. ${ }^{8,19-21}$ More recently, resonant $\mathrm{x}$-ray diffraction experiments have been performed at the $K$ edge ${ }^{7}$ and the $L_{2,3}$ edges of $\mathrm{Fe}^{10}{ }^{10}$ The studied $(0 k 0)$ reflections have the advantage that magnetic scattering does not contribute to the reflection intensities for the electric dipole (E1) transition. The experiments at the $K$ edge are sensitive to magnetoelectric multipoles of rank one and two and interpretation of data is based on different absorption events such as E1, magnetic dipole (M1) and electric quadrupole (E2). Experiments in the soft $\mathrm{x}$-ray regime ( $\mathrm{Fe} L_{2,3}$ edges) were interpreted in terms of the magnetoelectric charge (monopole) and quadrupole through an E1-M1 event. The basic concept of such an interpretation has been introduced in Ref. 8.

In the present study, we used resonant $\mathrm{x}$-ray diffraction at the Fe $K$ edge to observe the magnetoelectric multipole moments at space group and magnetic structure forbidden reflections. A single reflection of that type has previously been investigated with soft $\mathrm{x}$ rays ${ }^{10}$ but here we study several reflections by the magnetic field difference technique previously applied to the $K$ edge study of the space group allowed reflections. ${ }^{7}$ With such an approach, the strongest signals (charge and magnetic scattering) does not contribute to the observed signals. Clear nonzero differences are observed in 
the pre-edge regime and azimuthal angle scans give evidence for several multipole contributions to the scattering. The magnetoelectric behavior is evidenced by the sign change in the magnetic difference when interchanging the Friedel pair reflections $(0 k 0)$ and $(0 \bar{k} 0)$.

\section{EXPERIMENTS}

Stochiometric amounts of $\mathrm{Ga}_{2} \mathrm{O}_{3}(99.99 \%$ purity) and $\mathrm{Fe}_{2} \mathrm{O}_{3}$ ( $\geq 99.0 \%$ purity) were thoroughly ground together and then synthesized at $1200{ }^{\circ} \mathrm{C}$ for $20 \mathrm{~h}$ in air with an intermediate grinding after $10 \mathrm{~h}$. The powder was then compacted into a rod and sintered in a vertical tube furnace at $1350{ }^{\circ} \mathrm{C}$ for $30 \mathrm{~h}$ in air. Single crystals were grown under 8.5 bar oxygen at zoning rate of $2 \mathrm{~mm} / \mathrm{h}$. A single crystal was cut along the $\left(\begin{array}{lll}0 & 1 & 0\end{array}\right)$ surface and glued on to the copper sample holder mounted in a He flow cryostat reaching temperatures between 10 and $300 \mathrm{~K}$. Resonant x-ray diffraction experiments were performed at Diamond Light Source, on Beamline I16 (Materials \& Magnetism ${ }^{22}$ ). The horizontally polarized beam was monochromatized by a Si (111) channelcut monochromator. Experiments were performed with a focus of approximately $185 \times 35 \mu \mathrm{m}^{2}$ and $\mathrm{x}$-ray energies in the vicinity of the Fe $K$ edge $(7.1 \mathrm{keV})$. The magnetization of the sample was changed by mounting an electromagnet outside of the diffractometer and applying a field of $0.2 \mathrm{~T}$ when the sample was positioned with the $c$ axis (magnetization direction) collinear to the field. The corresponding azimuthal angle is a rotation about the reflection $\boldsymbol{\tau}=(0 \mathrm{k} 0)$ of $\psi=90^{\circ}$ for this configuration. Having the $c$ axis in the scattering plane corresponds to $\psi=90^{\circ}$. The experiments are performed in zero magnetic field (remanescence). To measure the second reflection of the Friedel pair, the sample was reglued upside down. This corresponds to a rotation of the crystal around the $c$ axis by $\pi$. All experiments were performed with incident $\mathrm{x}$-ray polarization normal to the plane of scattering $(\sigma$ polarization) and without analysis of the scattered x-ray polarization.

\section{RESULTS}

To obtain an energy scan of the space group forbidden reflection of type $(0 k 0)$ with $k$ odd in the vicinity of the $\mathrm{Fe} K$ edge, the azimuthal angle $\psi$ was scanned in the vicinity of the optimal angle, e.g., around $\psi=90^{\circ}$. A $\psi$ value in this region, without significant contributions from multiple scattering, was then selected for the energy scans, taken at fixed X-ray momentum transfer $(\mathbf{Q}=\boldsymbol{\tau})$. The energy scan of the (050) reflection is shown together with the fluorescence in Fig. 1. There are three clear features observed in the energy scan of the (050) reflection, located at approximately 7.113, 7.123 , and $7.129 \mathrm{keV}$. These features are robust against small variation in the azimuthal angle, indicative that they are not affected by remaining contributions of multiple-scattering peaks. The lowest lying peak is observed at the same energy as the pre-edge feature observed in the fluorescence spectra. The other two features are clearly appearing in the main edge regime, where the first derivative of the absorption $\mu(E)$ has maxima. This indicates that the main edge features are likely

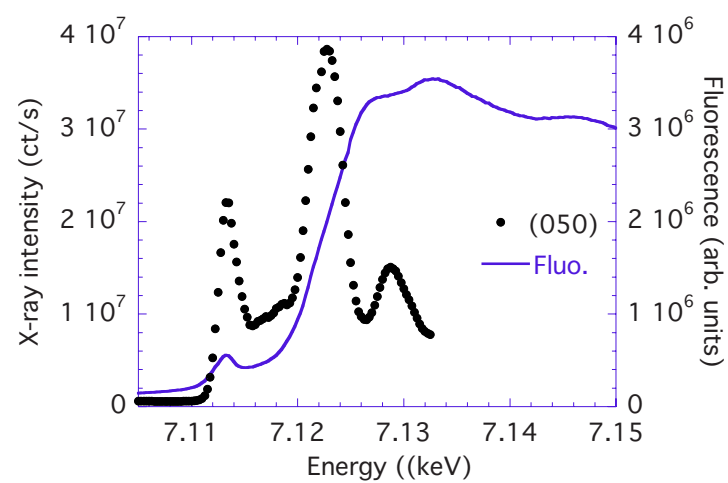

FIG. 1. (Color online) Peak maxima intensity of the space group forbidden (050) reflection of $\mathrm{GaFeO}_{3}$ in the vicinity of the $\mathrm{Fe} K$ edge taken at $100 \mathrm{~K}$ and $\psi=88.5^{\circ}$.

dominated by electric dipole-electric dipole (E1-E1) events, whereas the interpretation in the pre-edge regime is possibly more complex.

The two higher lying features are expected to be good candidates for Templeton and Templeton scattering (anisotropy of tensor of susceptibility) or often called orbital scattering. In case of $\mathrm{GaFeO}_{3}$, though, the term "orbital scattering" might be slightly misleading as the Fe $3 d^{5}$ shell is close to spherical. However, at the main edge, the empty $\mathrm{Fe} 4 p$ states are probed. These states may have a significant orbital moment and can therefore be strongly aspherical, leading to a significant scattering from the $4 p$ electric quadrupoles probed at the Fe $K$ edge. That the intensity is caused by the asphericity of the $4 p$ states can be seen from the azimuthal angle dependence of the three features shown in Fig. 2, which all follow a simple $\sin ^{2} \psi$ dependence, as predicted from the symmetry evaluation. ${ }^{10}$ Hybridization with oxygen $s$ states and covalent bonding would also allow the dipole E1-M1 transition to contribute. ${ }^{8}$ Note that there is a shift in the origin of $\psi$ between the soft $\mathrm{x}$-ray experiments ${ }^{10}$ and results presented here by $\pi / 2$.

The energy spectra of the intensity difference with having the sample in opposite magnetization states in the ferrimagnetic phase is shown in Fig. 3 together with its sum. The

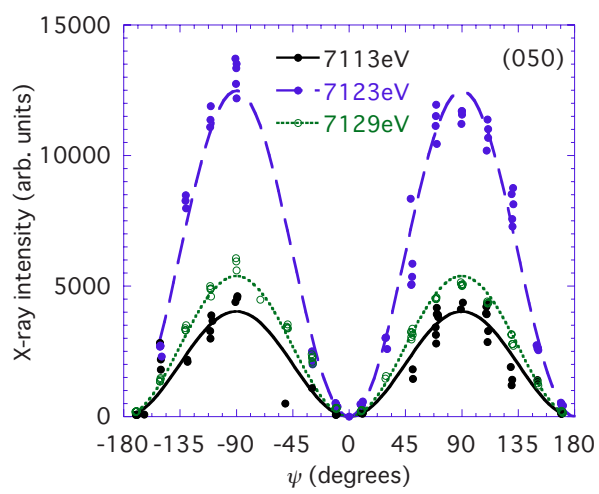

FIG. 2. (Color online) Azimuthal angle dependence of the (050) reflection taken at three different energies in the vicinity of the $\mathrm{Fe} K$ edge at $100 \mathrm{~K}$. The close lying points are taken at slight different azimuths at positions were the multiple-scattering contributions are weak. 


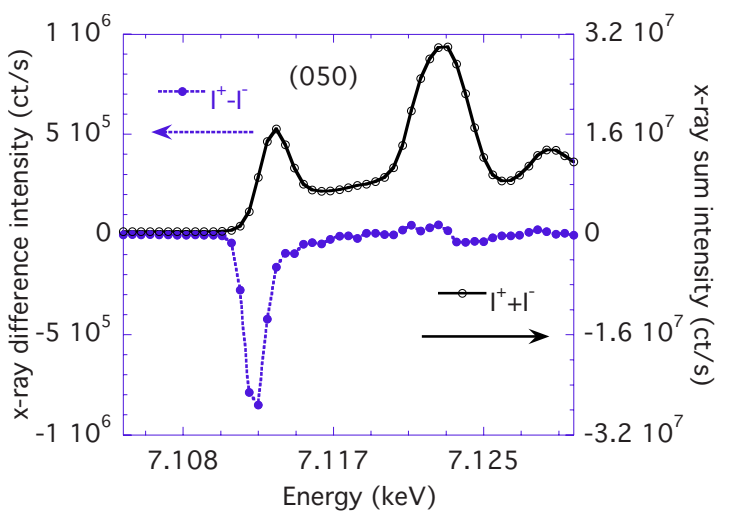

FIG. 3. (Color online) Sum (open circles) and difference (solid circles) of the energy-dependent $x$-ray intensity of the (050) reflection after the application of a magnetic field of $\pm 0.2 \mathrm{~T}$ taken at 110 K. Data are taken in remanescence.

difference intensity has clear minima at $7.112 \mathrm{keV}$, where the pre-edge feature of the sum has approximately its half height (a maximum in its first derivative). The absolute value of the difference intensity is at most $5 \%$ of the peak value. Only the pre-edge regime, where it is common to observe additional E1-E2 and E2-E2 and possibly E1-M1 events, a clear difference signal is observed. Note that from the soft $\mathrm{x}$-ray experiment on the forbidden (010) reflection and the symmetry analysis, ${ }^{10}$ we know already that scattering at reflections with $k$ odd $(0 k 0)$ can and do only occur in the rotated polarization channel $\sigma-\pi$ or $\pi-\sigma$.

The energy scans of three different Friedel pairs of the space group forbidden $(0 k 0)$ reflections are shown in Fig. 4. The spectra for $k= \pm 5$ are contaminated with one multiplescattering peak, which was identified through measurements of small variations in $\psi$. The energy dependence within each pair is significant different. Whereas there is always a clear pre-edge feature, though with different intensity, the spectral features at higher energies are not only different in their intensity ratio but also show features occurring at different energies. For example, we find three peaks in the E1-E1 dipole transition dominated regime for the (030) reflection at $7.113,7.1237$, and $7.129 \mathrm{keV}$, whereas the $(0 \overline{3} 0)$ reflection shows only two, at 7.122 and $7.1285 \mathrm{keV}$. As the azimuthal angle dependence indicated for the (050) reflection, that these features are of E1-E1 origin, it is unlikely to be different for the other obtained reflections. It is well known that Friedel pairs have equal intensity in nonresonant diffraction experiments. If however, the space group has no inversion symmetry, the Friedel pairs will get different intensity from each other at (or close to) resonance due to the fact that the individual complex contributions in the phase factor of the ions no longer cancel and are reversed within the individual Friedel pairs. The structure factor of a resonant space group forbidden reflection can be written as

$$
F(E)=\sum_{i} f_{i}(E) e^{i \tau \mathbf{R}_{i}}
$$

with $f^{\prime}(E)+i f^{\prime \prime}(E)$ as the real and imaginary parts of the resonant scattering factor and $\mathbf{R}_{i}$ the position of the $i$ th ion

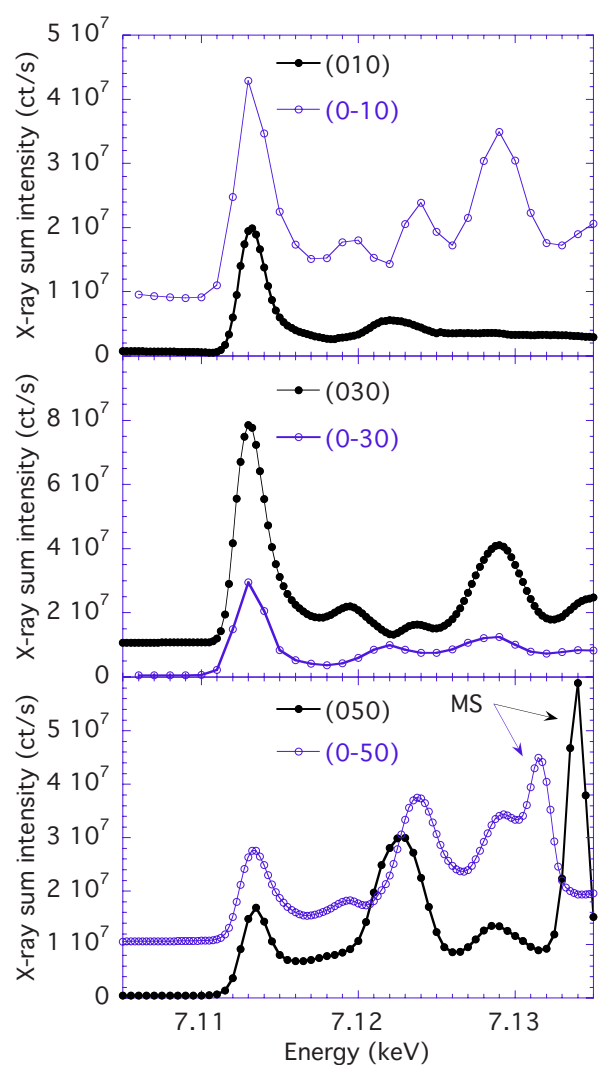

FIG. 4. (Color online) Energy dependence of space group forbidden $(0 k 0)$ reflections in $\mathrm{GaFeO}_{3}$ taken at $100 \mathrm{~K}$. Multiple scattering contributions are labeled "MS." The Friedel pairs were obtained by rotating the crystal around the crystal $c$ axis.

within the unit cell. It has been shown that for $\mathrm{GaFeO}_{3}$, only a single term, reflecting an electric quadrupole moment $\left\langle\mathbf{T}_{1}^{2}\right\rangle^{\prime \prime}$ contributes to the parity even E1-E1 scattering. A single damped harmonic-oscillator function gives $f^{\prime}(E)+i f^{\prime \prime}(E)$ $=f /\left(E_{i}-E_{g}-\hbar \omega+i \Gamma\right)$.

For any centrosymmetric crystal structure, the Friedel pairs have equal intensity (at any energy). Moreover, for any crystal symmetry, if the scattering arises only from a single resonance type, without interference of Thomson scattering, then the pairs must have identical spectra. Observation of different energy spectra for members of Friedel pairs of space group forbidden reflections therefore requires the presence of both a noncentrosymmetric structure and multiple resonance types. The present results therefore determine that the iron sites experience significantly different crystal fields.

The energy dependences of the difference of having the sample magnetized in the opposite directions for the Friedel pairs are shown in Fig. 5. Interestingly, for all reflections with positive $k$ the intensity differences are negative, and those for negative $k$ the intensity difference is always positive, at the energy with maximal difference. (Note that there is an arbitrary scale factor between the three reflections of positive $+k$ compared to those of $-k$.) The reversal between opposite signs of $k$ is a direct consequence of the magnetoelectric effect, as already investigated by resonant scattering techniques at the charge allowed reflections ${ }^{7}$ and the soft $\mathrm{x}$-ray resonant diffraction experiments. ${ }^{10}$ However, the inter- 


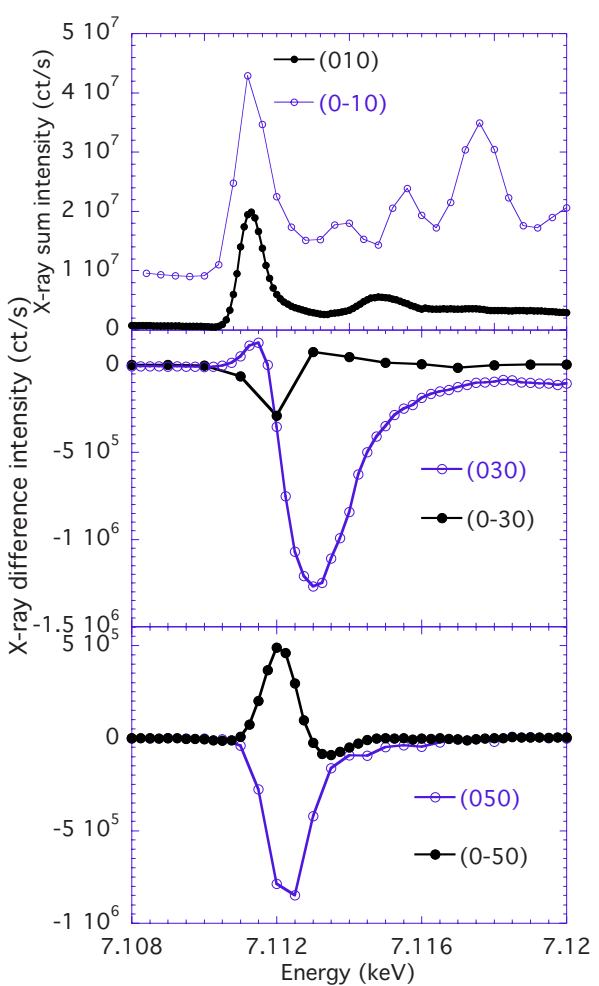

FIG. 5. (Color online) Energy dependence of the magnetization difference intensities of the space group forbidden $(0 k 0)$ reflections in $\mathrm{GaFeO}_{3}$ taken at $100 \mathrm{~K}$ and $\psi=-90^{\circ}$.

pretation is more rich, as can be seen from the maxima/ minima of the difference in the energy spectra which do not occur at the exact same energies for the Friedel pairs. Moreover, there is a clear change in sign in the energy dependence of the magnetization difference for both $k= \pm 3$ reflections. That the magnetic signal observed is coupled to the magnetic order of the system can be seen from the temperature dependence, which is shown in Fig. 6. It is evident that the difference signal disappears above $T_{\mathrm{C}}$.

To gain more information on the magnetoelectric type of scattering, we have collected azimuthal angle dependence of the difference intensities with different magnetization directions for the Friedel pair with $k= \pm 5$, which is shown in Fig.

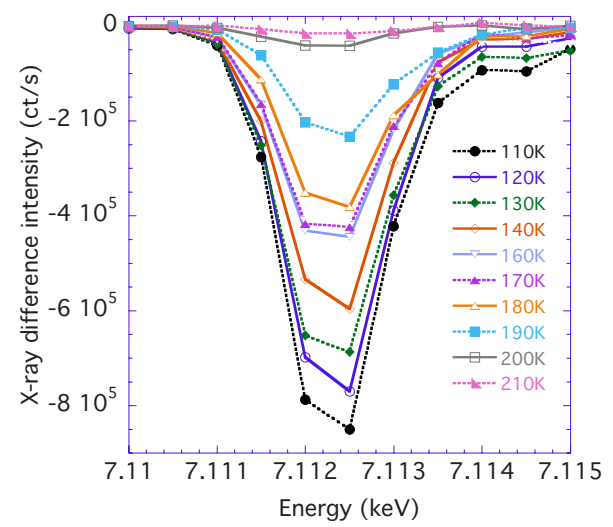

FIG. 6. (Color online) Magnetization difference intensities of the space group forbidden $(0 k 0)$ reflections in $\mathrm{GaFeO}_{3}$ for different temperatures taken at $\psi=-90^{\circ}$. The lines are guide to the eyes.

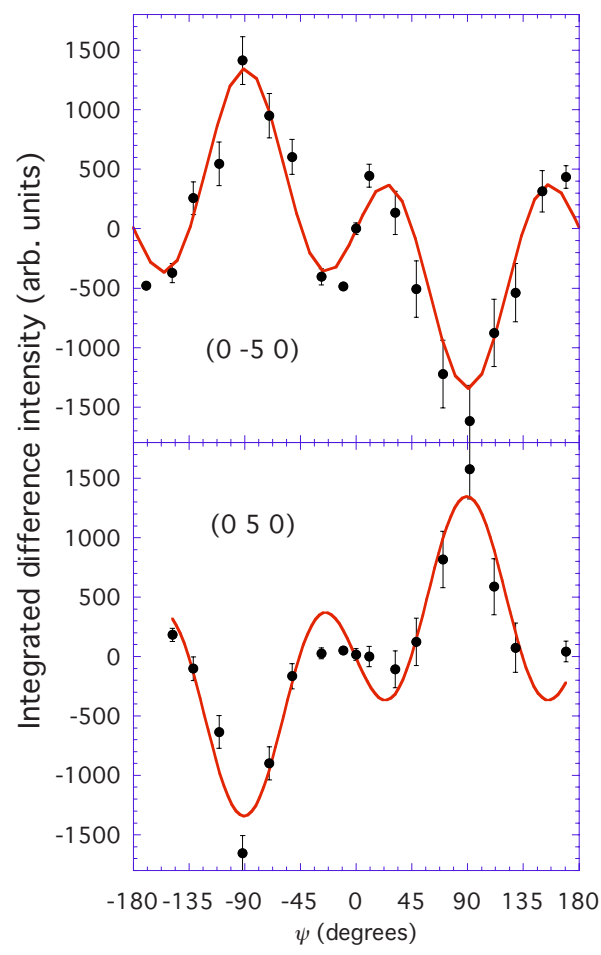

FIG. 7. (Color online) Azimuthal angle dependences of the difference $\mathrm{x}$-ray intensity for a magnetization sign change for the Friedel pair of $k= \pm 5$ in $\mathrm{GaFeO}_{3}$. The fit is based on a model of magnetoelectric multipole moments with an orbital contribution as explained in the text.

7. Again a clear inversion of the difference signal is observed at $\psi=-90^{\circ}$, at the position where the energy scan was taken. The azimuthal scan gives clear indication of more than one contribution to the scattering. It is not simply described by a sine or cosine function of the azimuthal angle $\psi$.

A more detailed analysis of the azimuthal angle dependence is based on the model successfully describing the data on the $(0 k 0)$ reflections taken at the $K$ edge of Fe for charge allowed reflections ( $k$ even) and for $k$ odd at the $\mathrm{Fe} L_{2,3}$ edges. ${ }^{8,10}$ For that purpose magnetoelectric multipole moments are additionally introduced named here $\mathbf{G}_{q}^{k}$. The magnetoelectric multipoles do change sign when changing the magnetization (are time odd) and they also change sign under inversion of the local space coordinates (parity odd). The electric quadrupole neither changes its sign when inverting time nor space since it is parity and time even. In this description here, we assume for simplicity that the energy position of the resonance is the same for all the terms with equal parity, which is a good approximation when considering a single parity even with a parity odd transition dominating the observed signals. Then the scattering amplitude can be written as ${ }^{8}$

$$
F \propto \sum_{j=\alpha, \beta} d_{j}(E)\left(C_{j}+M_{j}\right)
$$

for which the $d_{j}(E)$ represents the resonance (damped harmonic-oscillator function) and $C_{j}$ to the time even and $M_{j}$ to the time odd parts of the structure factor. The $j=\alpha \mathrm{com}-$ 
ponents can be assigned to be parity even and the $j=\beta$ components as parity odd. The individual terms in Eq. (2) are

$$
\begin{gathered}
C_{\alpha}=A t_{\alpha}(\psi) \sin (\psi), \\
M_{\alpha}=i B m_{\alpha}(\psi), \\
C_{\beta}=i B t_{\beta} \sin (\psi),
\end{gathered}
$$

and

$$
M_{\beta}=A m_{\beta}(\psi)
$$

From the definition above, $m$ represents magnetic terms and $t$ nonmagnetic terms, with $t_{\alpha}, m_{\alpha}$, and $m_{\beta}$ even functions of the azimuthal angle and $t_{\beta}$ independent of the angle. The first two terms [Eqs. (3a) and (3b)] are caused by parity even transitions such as E1-E1 or E2-E2 and the second two [Eqs. (3c) and (3d)] by either E1-M1 or E1-E2 transitions. The constants $A$ and $B$ are complex and solely defined by the phase factors of the $\mathrm{Fe}$ ion positions and the Miller index $k$ of the probed reflection as derived in Ref. 8, whereas $m$ and $t$ are purely real. A minimal model to describe the magnetic field difference reflection intensities can be based on the interference of two events and written as

$$
\Delta I \propto\left|\sum_{j=\alpha, \beta} d_{j}(E)\left(C_{j}+M_{j}\right)\right|^{2}-\left|\sum_{j=\alpha, \beta} d_{j}(E)\left(C_{j}-M_{j}\right)\right|^{2} .
$$

Equation (4) transforms to

$$
\begin{aligned}
\Delta I \propto 4\left|d_{\alpha}(E)\right|^{2} \sin (\psi)\left\{\left(A B^{*}\right)^{\prime \prime}\left[t_{\alpha} m_{\alpha}+|R(E)|^{2} t_{\beta} m_{\beta}\right]\right. \\
\left.+R(E)^{\prime}\left(|A|^{2} t_{\alpha} m_{\beta}+|B|^{2} m_{\alpha} t_{\beta}\right)\right\}
\end{aligned}
$$

with $R(E)=d_{\beta}(E) / d_{\alpha}(E)$. This approximation shows that there is an overall dependence of the difference intensity of the reflection going with $\sin \psi$, in accord with Fig. 7. Changing from $k$ to $-k$, leads to a sign change in the parity even terms $t_{\alpha}$ and $m_{\alpha}$ and the complex phase factors $A$ and $B$ will transform to $A^{*}$ and $B^{*}$. Note $k \rightarrow-k$ corresponds to a flipping of the sample upside down (in our case a rotation around the $c$ axis by $\pi$ ) and is not equivalent to an inversion operation. As already pointed out, the tensorial contribution of the E1-E1 event has a single quadrupole and $t_{\alpha}$ does not depend on $\psi$ and $m_{\alpha}=0$ because the magnetization lies along the crystal $c$ axis. For E1-M1 the formula can be easily extracted from Ref. 10 , and $t_{\beta}$ contains simply the polar moment $\left\langle U_{0}^{1}\right\rangle$ and $m_{\beta}$ the magnetic charge (monopole) $\left\langle G_{0}^{0}\right\rangle$ and the two components of the magnetoelectric quadrupole $\left\langle G_{0}^{2}\right\rangle$ and $\left\langle G_{2}^{2}\right\rangle^{\prime}$, which was determined at the Fe $L_{2,3}$ edges. Note that there is an origin shift in $\psi$ of $\pi / 2$ between this study and Ref. 10. Considering the E1-E2 event for the pre-edge to dominate the parity odd event, $t_{\beta}$ will contain several polar multipoles, including dipolar, quadrupolar, and octopolar contributions, but $t_{\beta}$ will still remain independent on $\psi$. For the magnetoelectric contribution $m_{\beta}(\psi)$, the parity and time odd term, there is no monopole contribution $\left\langle G_{0}^{0}\right\rangle$. Instead, because it is a higher order transition, a component of the magnetoelectric octopole appears and the explicit form of $M_{\beta}(\psi)$ in Eq. (3) is written following Ref. 8 as

$$
\begin{aligned}
M_{\beta}(\psi)= & \frac{A}{\sqrt{5}}\left\{-\cos ^{2} \theta \cos (2 \psi)+\frac{1}{2}[3 \cos (2 \theta)-1]\right\}\left\langle G_{0}^{2}\right\rangle^{\prime \prime} \\
& +\frac{4 A}{\sqrt{30}}\left\{\frac{1}{2} \cos ^{2} \theta \cos (2 \psi)+\frac{3}{4}[3 \cos (2 \theta)-1]\right\}\left\langle G_{0}^{2}\right\rangle^{\prime} \\
& -\frac{8 A}{\sqrt{15}} \cos ^{2} \theta \cos (2 \psi)\left\langle G_{2}^{3}\right\rangle^{\prime \prime} .
\end{aligned}
$$

\section{DISCUSSION}

The observed differences of Friedel pairs of space group forbidden reflections and its dependence on the azimuthal angle $\psi$ can be well described by a simple atomic model of interference between a parity even and a parity odd event. The difference to the interpretation of the resonant diffraction study in the soft $\mathrm{x}$-ray regime $\left(L_{2,3}\right.$ edges $)$ depends strongly on which transition is indeed dominating in the $\mathrm{Fe} K$ pre-edge region. If the E1-E1 and E1-M1 transitions would dominate, the experiment would have observed the magnetoelectric quadrupole and possibly its monopole. However, these multipoles would represent the properties of the Fe $4 p$ states. That such multipoles can indeed have nonzero expectation values has been recently and explicitly shown by use of model wave functions. ${ }^{23}$ They are based on the overlap of parity even and parity odd wave functions of the open Fe $3 d$ shell with those of the neighboring $2 p$ shell. In contrast to the $L$ edge experiments the time and parity even contribution from the E1-E1 is expected to be much stronger than the E1-M1 magnetoelectric term and the polar term. This is due to two reasons. First, the $4 p$ shell electric quadrupole directly relates to the $\mathrm{O} 2 p, \mathrm{Fe} 4 p$ overlap and can be large, whereas the $3 d^{5}$ shell is almost spherical having a small electric quadrupole only. Second, because the $\mathrm{Fe} 4 p$ states are probed, the magnetic (magnetoelectric) signal is related to an induced weak magnetic moment in the $4 p$ shell, with correspondingly weak magnetoelectric signals. In addition, one would expect significant magnetic contributions to the main edge regime too because both transitions are of dipole origin. No magnetic contribution is observed at the main edge. Even though the E1-M1 transition has been used to describe the interference (with the E1-E2 transition) observed in the energy scan of the space group allowed reflections $k$ even, it is more likely that the main contributions observed here are coming from the interference of the E1-E1 and E1-E2 terms. In this case, the experiment confirms clearly the observation of significant contributions of the magnetoelectric multipole moments of higher rank. These experiments therefore give further confidence in the occurrence of significant magnetoelectric multipole moments in the $3 d$ shell of $\mathrm{Fe}$ and are in good agreement with those of the Fe $L_{2,3}$ edges probing directly the Fe $3 d$ shell. Unfortunately, even though $m_{a}$ $=0$, the individual sizes of the three contributing magnetoelectric multipole moments cannot be extracted. A further comparison with the $K$ edge experiments on the space group 
allowed reflection is not possible, as the changing $k$ even with $k$ odd leads to different selection rules for the magnetoelectric and polar multipoles. The $k$ even experiment is sensitive to the toroidal moment (anapole or magnetoelectric dipole) and also to the magnetoelectric quadrupole. But the quadrupole term have different projections compared to the $k$ odd reflections. Another important difference concerns the fact that at these space group allowed reflections, a ferro-type order is probed, in contrast to the space group forbidden $k$ odd reflections, where the antiferro-type order of these strange multipoles are probed.

The observation of magnetoelectric multipole moments is an excellent way to test the wave functions of magnetic ions on sites with no inversion symmetry. Theoretical attempts in describing the observations have already been done for the $k$ even reflections $7,8,21$ and for the $\mathrm{X}$-ray absorption experiments. ${ }^{8,24}$ Understanding the interaction of magnetic structure and ferroelectricity in multiferroics on the level of atomic wave functions is certainly a challenge today. The observation of magnetoelectric multipole moments can certainly contribute in this, as they represent the interesting overlap of these wave functions responsible for the magnetoelectric coupling. Further experimental efforts in finding systems, where individual magnetoelectric multipoles can be singled out as well as further theoretical effort in describing the energy dependence of these multipoles will be important for understanding the magnetoelectric effect on the atomic level.

\section{CONCLUSIONS}

Resonant x-ray diffraction experiments on space group forbidden reflections of type $(0 k 0)$ with $k$ odd taken in the vicinity of the Fe $K$ edge are presented on magnetoelectric $\mathrm{GaFeO}_{3}$. The observed $\sin \psi$ dependence on the azimuthal angle of the different spectra features indicates a dominance of the E1-E1 transition and can be ascribed to the observation of an electric quadrupole moment. The different spectral shapes collected for the Friedel pairs give indications of interference effects due to contributions from different Fe sites. It is shown that collecting the difference intensity with magnetization parallel or antiparallel to the $c$ axis in the ferrimagnetic phase test directly magnetoelectric multipole moments, which are both parity and time odd, and disappear at the ferrimagnetic order temperature. The azimuthal angle dependence and the sign change in the magnetic signal when going from $(0 k 0)$ to $(0 \bar{k} 0)$ reflections can be understood by the interference of the electric quadrupole with that of magnetoelectric quadrupole and octupole moments in the frame work of E1-E1 and E1-E2 transitions.

\section{ACKNOWLEDGMENTS}

We acknowledge the help of G. Nisbet during beamtime at I16. This work was supported by Swiss National Science Foundation NCCR MaNEP Project.
${ }^{1}$ T. Kimura, T. Goto, H. Shintani, K. Ishizaka, T. Arima, and Y. Tokura, Nature (London) 426, 55 (2003).

${ }^{2}$ N. Hur, S. Park, P. A. Sharma, J. S. Ahn, S. Guha, and S.-W. Cheong, Nature (London) 429, 392 (2004).

${ }^{3}$ Y. Yamasaki, H. Sagayama, T. Goto, M. Matsuura, K. Hirota, T. Arima, and Y. Tokura, Phys. Rev. Lett. 98, 147204 (2007).

${ }^{4}$ Y. Bodenthin, U. Staub, M. García-Fernández, M. Janoschek, J. Schlappa, E. I. Golovenchits, V. A. Sanina, and S. G. Lushnikov, Phys. Rev. Lett. 100, 027201 (2008).

${ }^{5}$ B. B. Van Aken, J. P. Rivera, H. Schmid, and M. Fiebig, Nature (London) 449, 702 (2007).

${ }^{6}$ S. W. Lovesey, J. Fernández-Rodríguez, J. A. Blanco, D. S. Sivia, K. S. Knight, and L. Paolasini, Phys. Rev. B 75, 014409 (2007).

${ }^{7}$ T. Arima, J. H. Jung, M. Matsubara, M. Kubota, J. P. He, Y. Kaneko, and Y. Tokura, J. Phys. Soc. Jpn. 74, 1419 (2005).

${ }^{8}$ S. W. Lovesey, K. S. Knight, and E. Balcar, J. Phys.: Condens. Matter 19, 376205 (2007).

${ }^{9}$ V. Scagnoli and S. W. Lovesey, Phys. Rev. B 79, 035111 (2009).

${ }^{10}$ U. Staub, Y. Bodenthin, C. Piamonteze, M. García-Fernández, V. Scagnoli, M. Garganourakis, S. Koohpayeh, D. Fort, and S. W. Lovesey, Phys. Rev. B 80, 140410(R) (2009).
${ }^{11}$ J. P. Remeika, J. Appl. Phys. 31, S263 (1960).

${ }^{12}$ G. T. Rado, Phys. Rev. Lett. 13, 335 (1964).

${ }^{13}$ R. B. Frankel, N. A. Blume, S. Foner, A. J. Freemann, and M. Schieber, Phys. Rev. Lett. 15, 958 (1965).

${ }^{14}$ E. A. Wood, Acta Crystallogr. 13, 682 (1960).

${ }^{15}$ T. Arima, D. Higashiyama, Y. Kaneko, J. P. He, T. Goto, S. Miyasaka, T. Kimura, K. Oikawa, T. Kamiyama, R. Kumai, and Y. Tokura, Phys. Rev. B 70, 064426 (2004)

${ }^{16}$ J. Y. Kim, T. Y. Koo, and J. H. Park, Phys. Rev. Lett. 96, 047205 (2006).

${ }^{17}$ J. H. Jung, M. Matsubara, T. Arima, J. P. He, Y. Kaneko, and Y. Tokura, Phys. Rev. Lett. 93, 037403 (2004).

${ }^{18}$ M. Kubota, H. Nakao, Y. Murakami, Y. Taguchi, M. Iwama, and Y. Tokura, Phys. Rev. B 70, 245125 (2004).

${ }^{19}$ J. I. Igarashi and T. Nagao, Phys. Rev. B 80, 054418 (2009).

${ }^{20}$ J. Igarashi and T. Nagao, J. Phys. Soc. Jpn. 79, 014705 (2010).

${ }^{21}$ S. Di Matteo and Y. Joly, Phys. Rev. B 74, 014403 (2006).

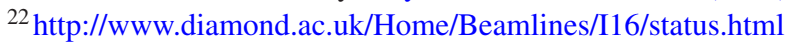

${ }^{23}$ S. W. Lovesey and V. Scagnoli, J. Phys.: Condens. Matter 21, 474214 (2009).

${ }^{24}$ M. Kubota, T. Arima, Y. Kaneko, J. P. He, X. Z. Yu, and Y. Tokura, Phys. Rev. Lett. 92, 137401 (2004). 